\title{
DIAGNÓSTICO DE DOENÇAS METABÓLICAS DO SISTEMA NERVOSO DA INFÂNCIA POR EXAME ULTRA-ESTRUTURAL DE TECIDO NÃO CEREBRAL
}

\author{
SÉRGIO ROSEMBERG*
}

\begin{abstract}
RESUMO - Se bem que as análises bioquímica e de genética molecular sejam os métodos mais precisos para o diagnóstico de doenças metabólicas, os estudos morfológicos permanecem um método diagnóstico muito importante principalmente em países como o Brasil, onde os laboratórios clínicos não estão aptos a realizar a maior parte dos exames requeridos para o reconhecimento destas doenças. Ainda, o exame anátomo-patológico é o único método diagnóstico para certas doenças cujo defeito metabólico é desconhecido tais como as lipofuscinoses ceróides, a distrofia neuroaxonal infantil e a doença de Lafora. Apresentamos nossa experiência com análise ultra-estrutural em 582 exames de conjuntiva ocular $(n=320)$, pele $(n=92)$ ou nervo periférico $(n=170)$ realizados entre 1975 e 1996, em 486 crianças. Em 112 casos, o exame revelou-se anormal. Em 59, o exame ultra-estrutural isoladamente fez o diagnóstico. Em 29 casos, o exame foi menos específico, tendo o diagnóstico final sido feito através da combinação da análise clínica e patológica. Nos 24 casos restantes, o diagnóstico genérico de uma mucopolissacaridose foi feito em 8 casos, de oligossacaridose em 4 e o de gangliosidose GM2 em 8. Sempre que a análise bioquímica pôde ser procedida, o diagnóstico ultra-estrutural foi confirmado. Estes resultados sublinham a importância do exame ultra-estrutural em tecidos não cerebrais em muitas doenças metabólicas, sobretudo quando testes bioquímicos não podem ser realizados.
\end{abstract}

PALAVRAS-CHAVE: doenças metabólicas, ultra-estrutura, diagnóstico anátomo-patológico.

\section{Diagnosis of metabolic diseases of the nervous system in children through ultrastructural analysis of non cerebral tissue.}

ABSTRACT - Although biochemical and molecular genetic analysis are the most precise methods for the diagnosis of metabolic diseases, morphological studies remain a very important diagnostic method mainly in countries like Brazil, where clinical laboratories are unable to perform most of the exams required for the diagnosis of these diseases. Moreover, pathologic evaluation is the single diagnostic method for some disorders whose metabolic defect is unknown such as neuronal ceroid-lipofuscinosis, infantile neuroaxonal dystrophy or Lafora disease. We present our experience with ultrastructural analysis in 582 exams of ocular conjunctiva $(n=320)$, skin $(n=92)$ or peripheral nerve $(n=170)$ performed between 1975 and 1996, in 486 children. In 112 cases there were definit ultrastructural changes. In 59 cases, the sole ultrastructural exam allowed the diagnosis. In 29 , the changes were less specific, and the final diagnosis was performed by a combination of clinical and pathological analysis. In the remaining 24 cases, a generic diagnosis of mucopolysaccharidosis was done in 8 cases, oligosaccharidosis in 4 cases and GM2 gangliosidosis in 12 cases. Whenever a biochemical test was performed in overseas laboratories, the initial diagnosis was confirmed. These results stress the importance of ultrastructural analysis in non-cerebral tissues for the diagnosis of many metabolic disorders mainly when biochemical tests cannot be performed.

KEY WORDS: metabolic diseases, ultrastructural exam, pathological analysis.

Se bem que a análise bioquímica ou exames de genética molecular sejam os métodos diagnósticos mais precisos para a caracterização das doenças metabólicas, estudos morfológicos permanecem um instrumento muito importante para o diagnóstico destas entidades, sobretudo em países como o

*Professor Associado do Departamento de Patologia, Disciplina de Neuropatologia, Faculdade de Medicina da Universidade de São Paulo e Professor Titular do Departamento de Pediatria, Disciplina de Neuropediatria, Faculdade de Ciências Médicas da Santa Casa de São Paulo. Aceite: 22-abril-1998.

Dr. Sérgio Rosemberg - Av. Dr. Arnaldo 455 - 01246-903 São Paulo SP - Brasil. 
Brasil onde a maioria, senão todos, os laboratórios de análise clínica não estão capacitados para a execução de grande parte dos exames necessários ${ }^{1}$. Assim, o diagnóstico laboratorial da quase totalidade das lisossomopatias, peroxissomopatias, amino e organoacidopatias ou mitocondriopatias é presuntivo, repousando sobre dados clínicos e outros exames laboratoriais, como neuro-imagem ou análises bioquímicas inespecíficas. Nestes casos, o exame morfológico, sobretudo através da análise ultra-estrutural de tecido não cerebral, como pele, conjuntiva ocular ou nervo periférico, pode ter relevante importância, permitindo, pelo menos, a caracterização definitiva de uma doença metabólica e maior aproximação diagnóstica. Ainda, a análise morfológica é o único diagnóstico em algumas doenças metabólicas cuja caracterização bioquímica é desconhecida, como é o caso das lipofuscinoses ceróides, da distrofia neuroaxonal infantil ou da doença de Lafora²

O objetivo deste estudo é o de apresentar a experiência de realização do diagnóstico de doenças metabólicas do sistema nervoso da infância através de exame ultra-estrutural de tecido não cerebral das Disciplinas de Neuropediatria e de Neuropatologia dos Departamentos de Pediatria e de Patologia das Faculdades de Ciências Médicas da Santa Casa de São Paulo e de Medicina da Universidade de São Paulo, respectivamente.

\section{MATERIAL E MÉTODO}

Quinhentos e oitenta e dois exames ultra-estruturais provenientes de 486 crianças suspeitas de serem portadoras de doenças metabólicas do sistema nervoso provenientes em sua maioria das clínicas neuropediátricas do Hospital das Clínicas da Faculdade de Medicina da Universidade de São Paulo (FMUSP) e Hospital da Santa Casa de São Paulo foram realizados entre 1975 e 1996. Alguns pacientes foram biopsiados mais de uma vez, para obtenção de tecidos diferentes, quando necessário para elucidação do diagnóstico final. O material, após preparação de rotina para microscopia eletrônica, foi examinado no Departamento de Patologia da FMUSP. Dos 582 exames, 320 corresponderam a conjuntiva ocular, 92 , a pele e 170 , a nervo periférico.

\section{RESULTADOS}

Observaram-se alterações ultra-estruturais em 112 casos. Destes, em 59 casos, a análise ultra-estrutural isolada permitiu o diagnóstico definitivo: leucodistrofia metacromática em 22; forma infantil tardia de lipofuscinose neuronal (doença de Bielschowsky) em 15; doença de Krabbe em 6; doença de Niemann-Pick tipo C em 3; doença de Gaucher tipo II em 1; distrofia neuroaxonal infantil em 10; doença de Lafora em 2.

Em 29 casos, as alterações ultra-estruturais foram menos específicas. Nestes casos, o diagnóstico foi feito através da combinação dos achados morfológicos, clínicos e de outros exames laboratoriais tais como exame radiológico do esqueleto, mielograma, biomicroscopia ocular, etc.: gangliosidose GM1 forma A em 10 casos; doença de Hurler em 6 casos; doença de Hunter em 4 casos; doença de Sanfilippo A?, B?, C?, D? em 4 casos; doença de Scheie em 1 caso; doença de Maroteaux-Lamy em 1 caso; doença de Austin em 1 caso e doença de Fabry em 2 casos.

Nos 24 casos restantes, um diagnóstico genérico de mucopolissacaridose foi feito em 8 casos, de oligossacaridose em 4 casos e de gangliosidose GM2 em 12 casos.

Sempre que um teste enzimático foi realizado - o que ocorreu sempre em laboratórios estrangeiros - o diagnóstico inicial foi confirmado, não tendo sido observados falsos resultados negativos ou positivos: leucodistrofia metacromática em 16 casos, doença de Krabbe em 4, gangliosidose GM1 em 4, doença de Sanfilippo tipo C em 4, doença de Tay-Sachs em 3 e doença de Sandhoff em 5.

\section{DISCUSSÃO}

O exame ultra-estrutural de tecido não cerebral permanece um valioso método diagnóstico das doenças metabólicas do sistema nervoso, sobretudo quando as dosagens enzimáticas específicas ou análises de genética molecular não podem ser realizadas.

Em três doenças do grupo, nas quais as bases bioquímicas permanecem ignoradas, esse exame é o único que permite o diagnóstico de certeza. Assim, os achados de corpos curvilíneos (Fig 1), de 


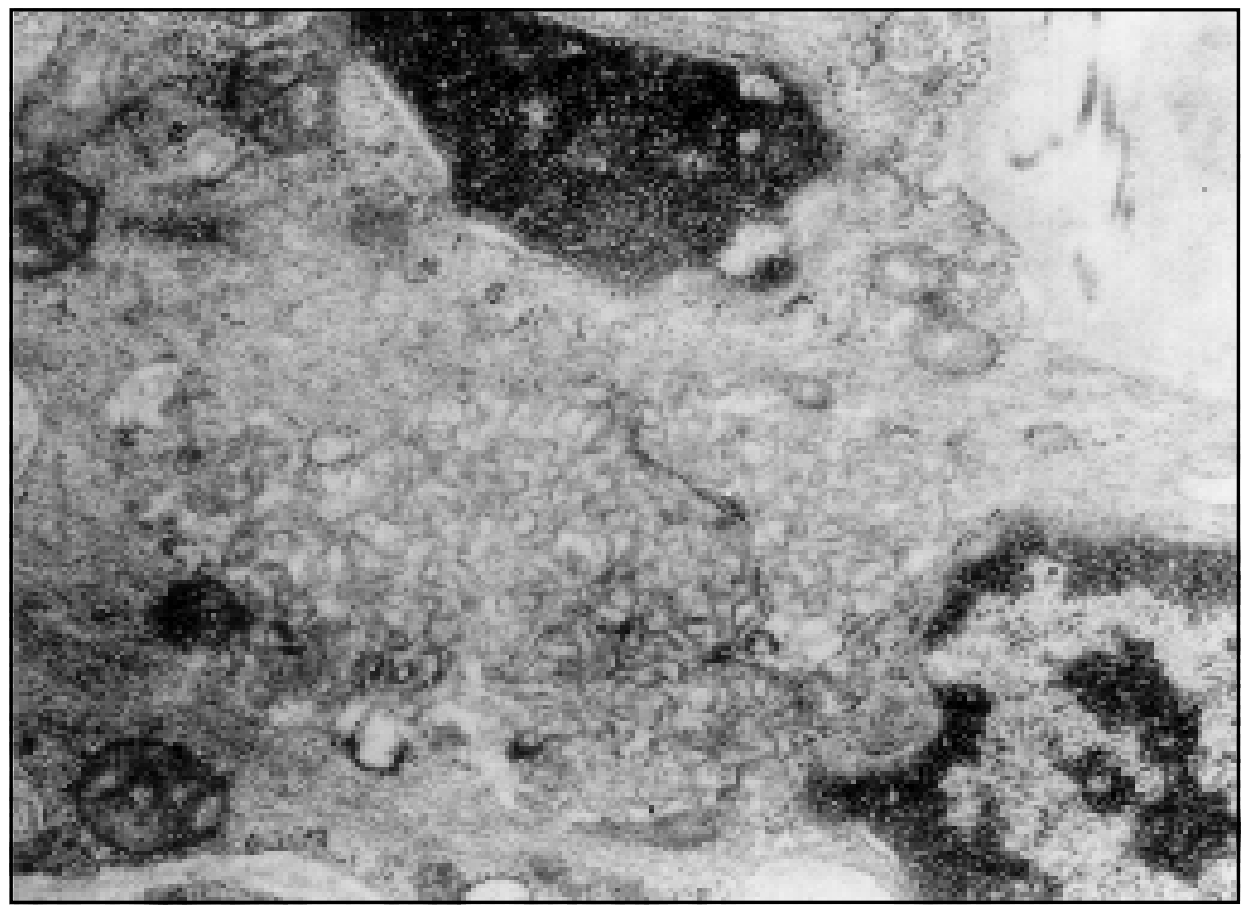

Fig 1. Doença de Bielschowsky. Corpos curvilíneos em célula endotelial de conjuntiva.

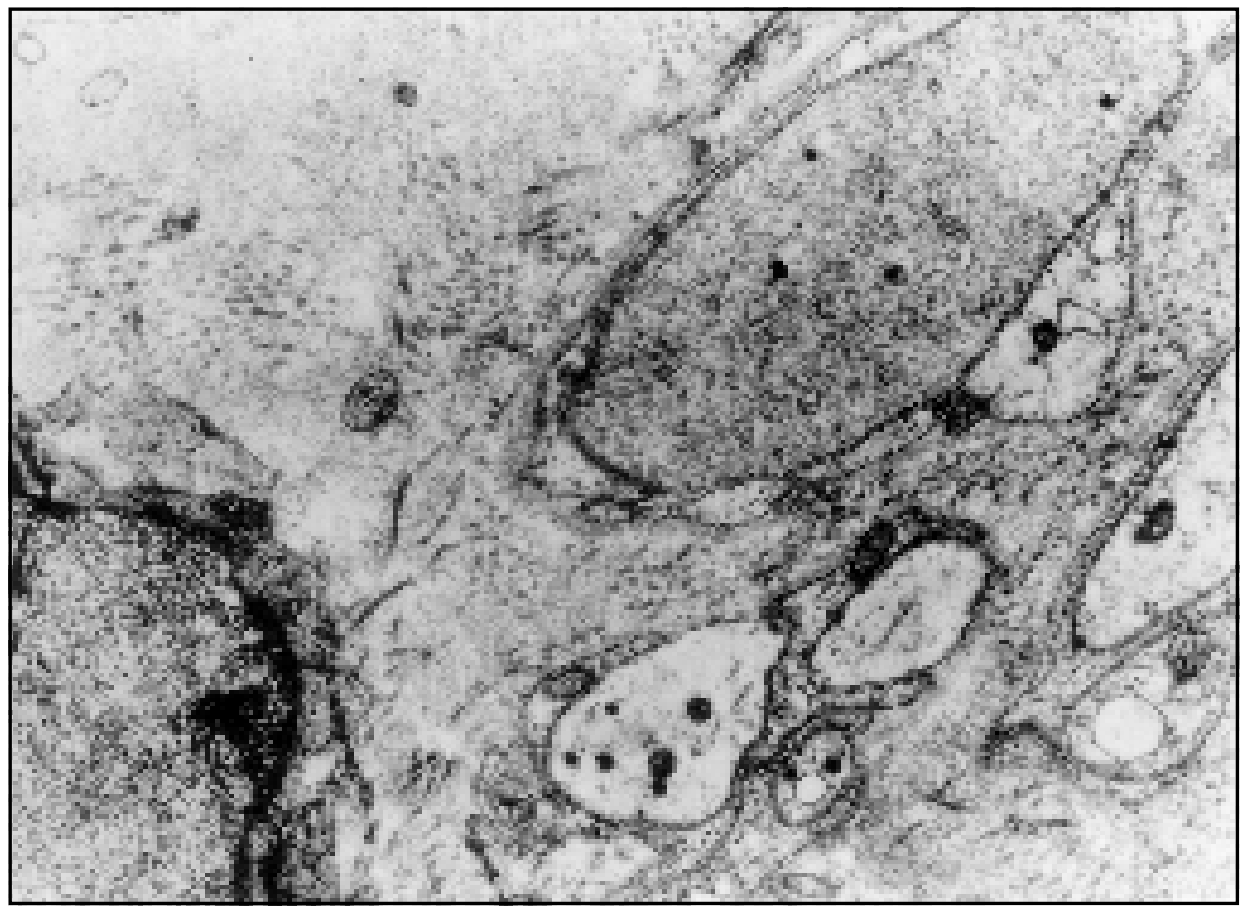

Fig 2. Distrofia neuroaxonal infantil.Axônio amielínico distrófico em um nervo conjuntival. 


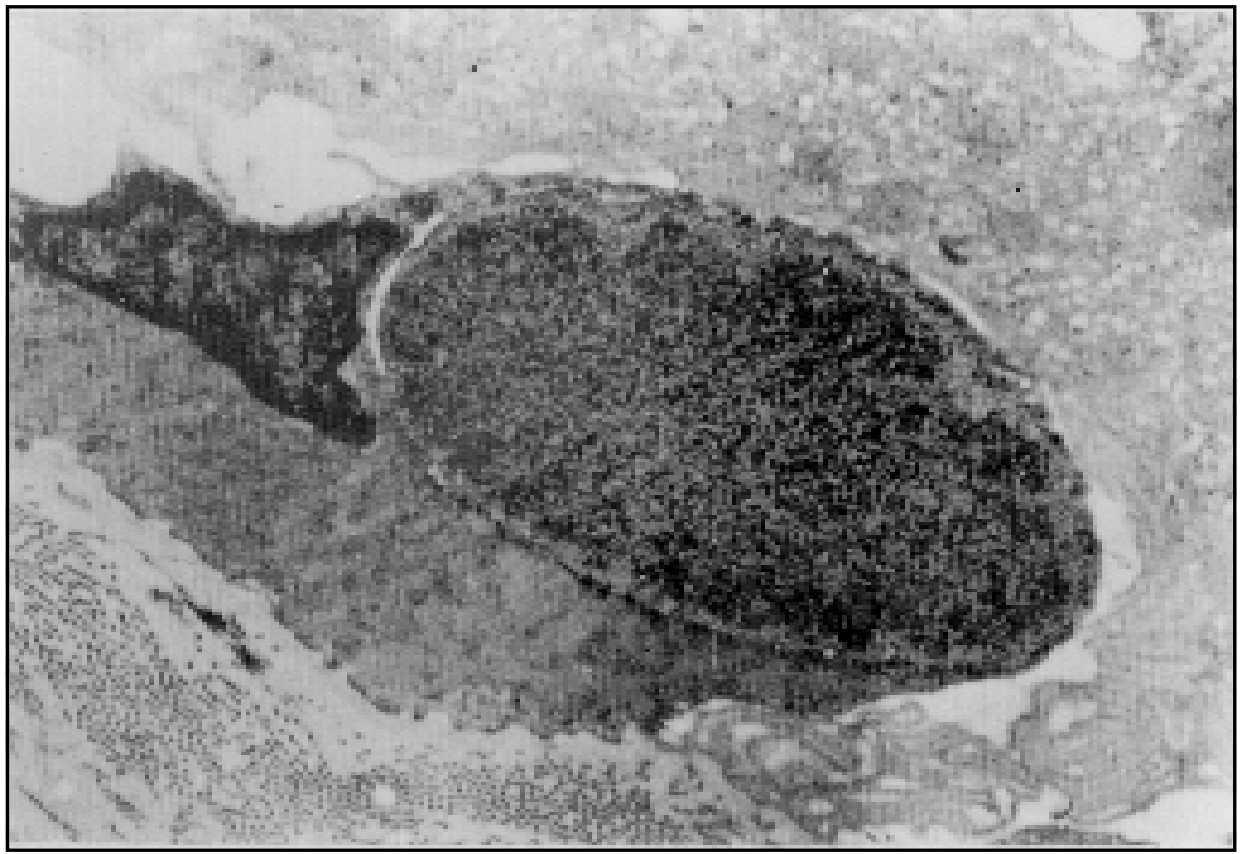

Fig 3. Doença de Lafora. Corpúsculo em citoplasma de célula mio-epitelial de pele axilar.

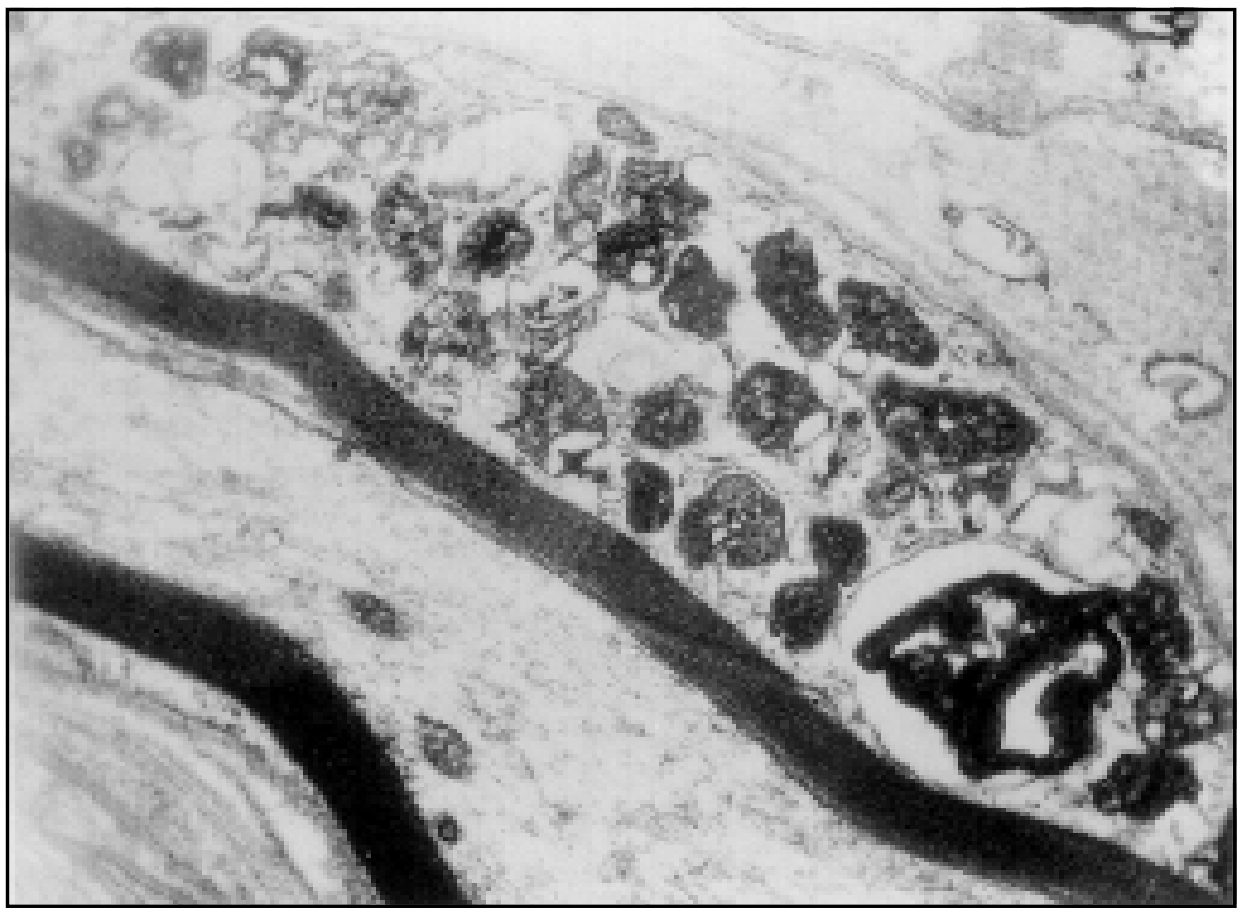

Fig 4. Leucodistrofia metacromática. Inclusões citoplasmáticas em célula de Schwann de nervo sural. 


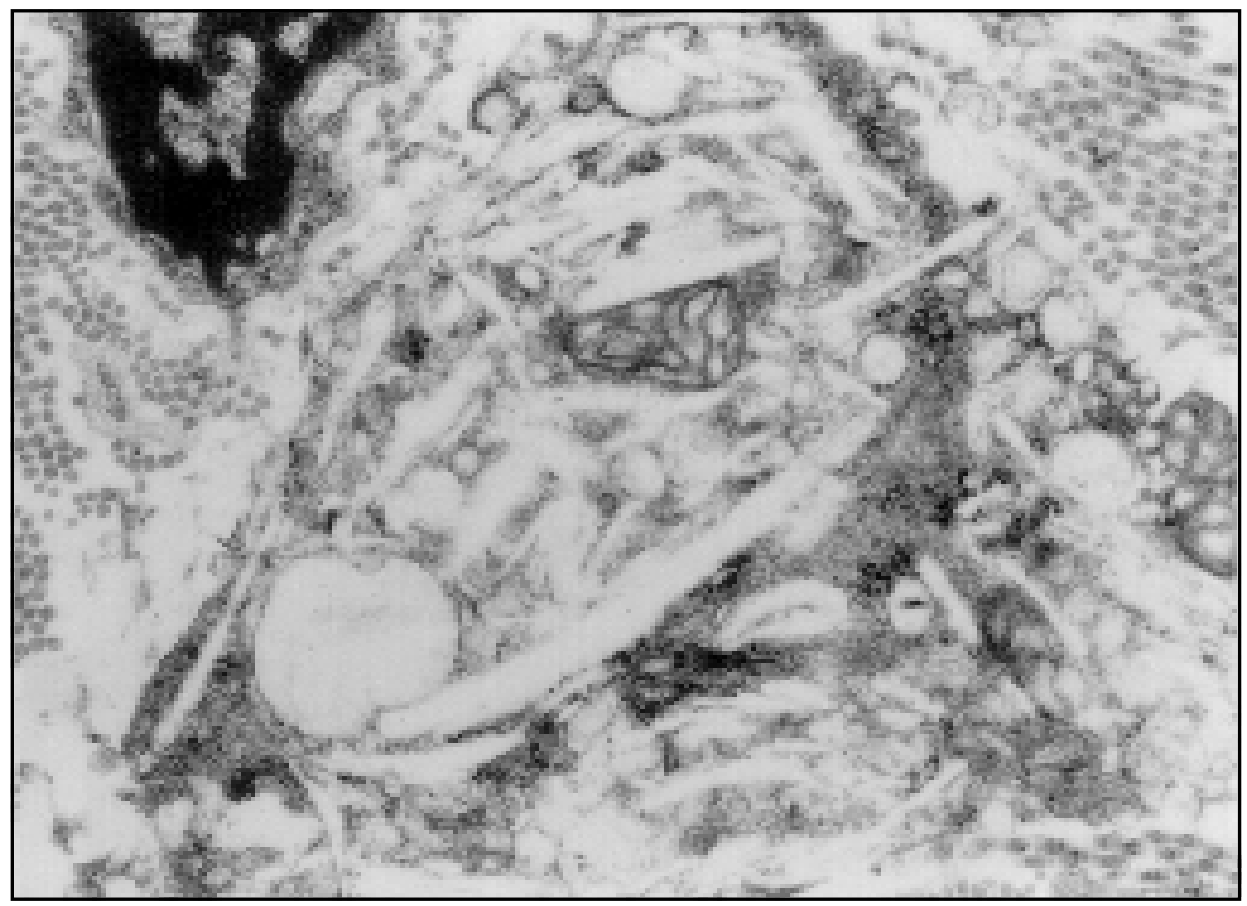

Fig 5. Doença de Krabbe. Inclusões cristaliformes em macrófago de conjuntiva.

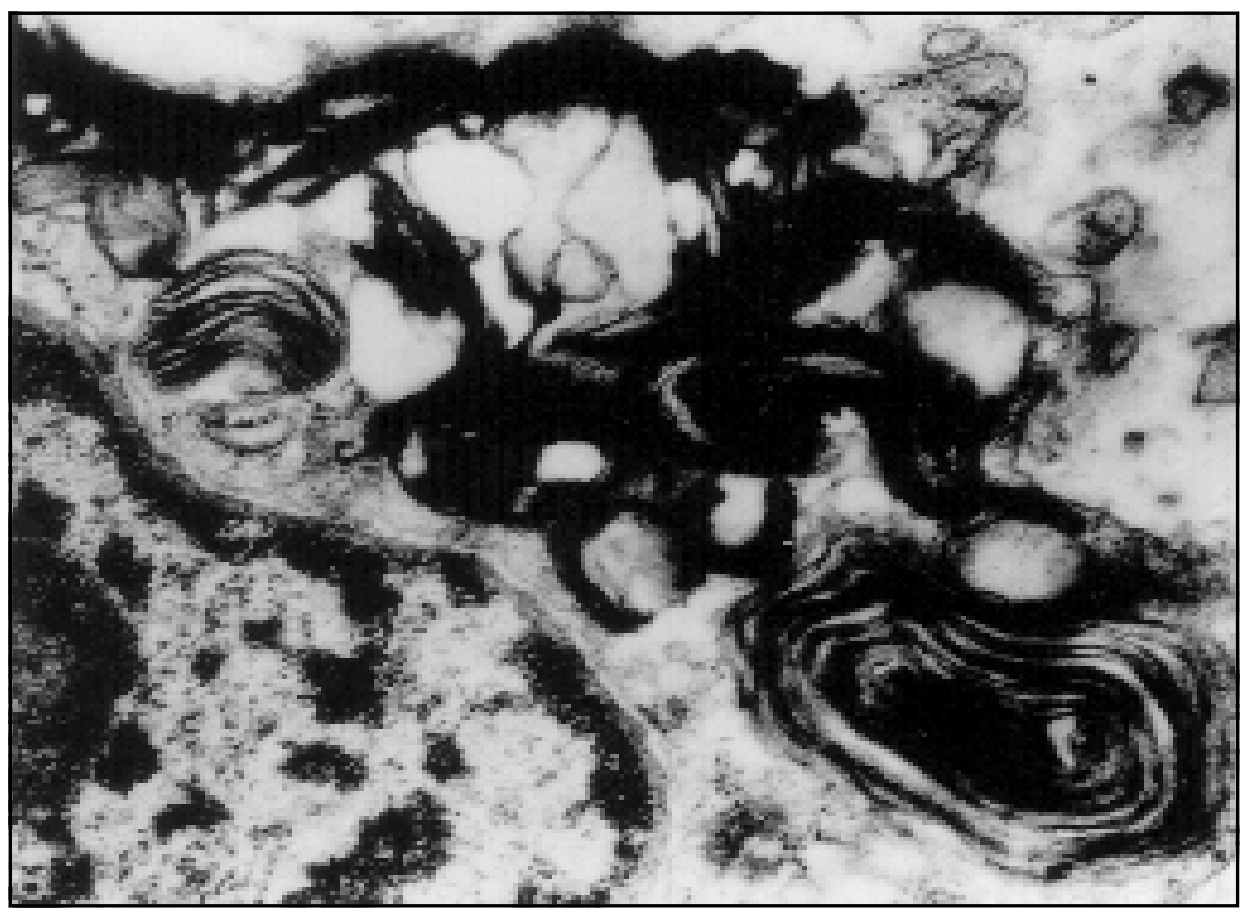

Fig 6. Doença de Niemann-Pick. Corpúsculos membrano-citoplasmáticos-símiles em fibroblasto de conjuntiva. 


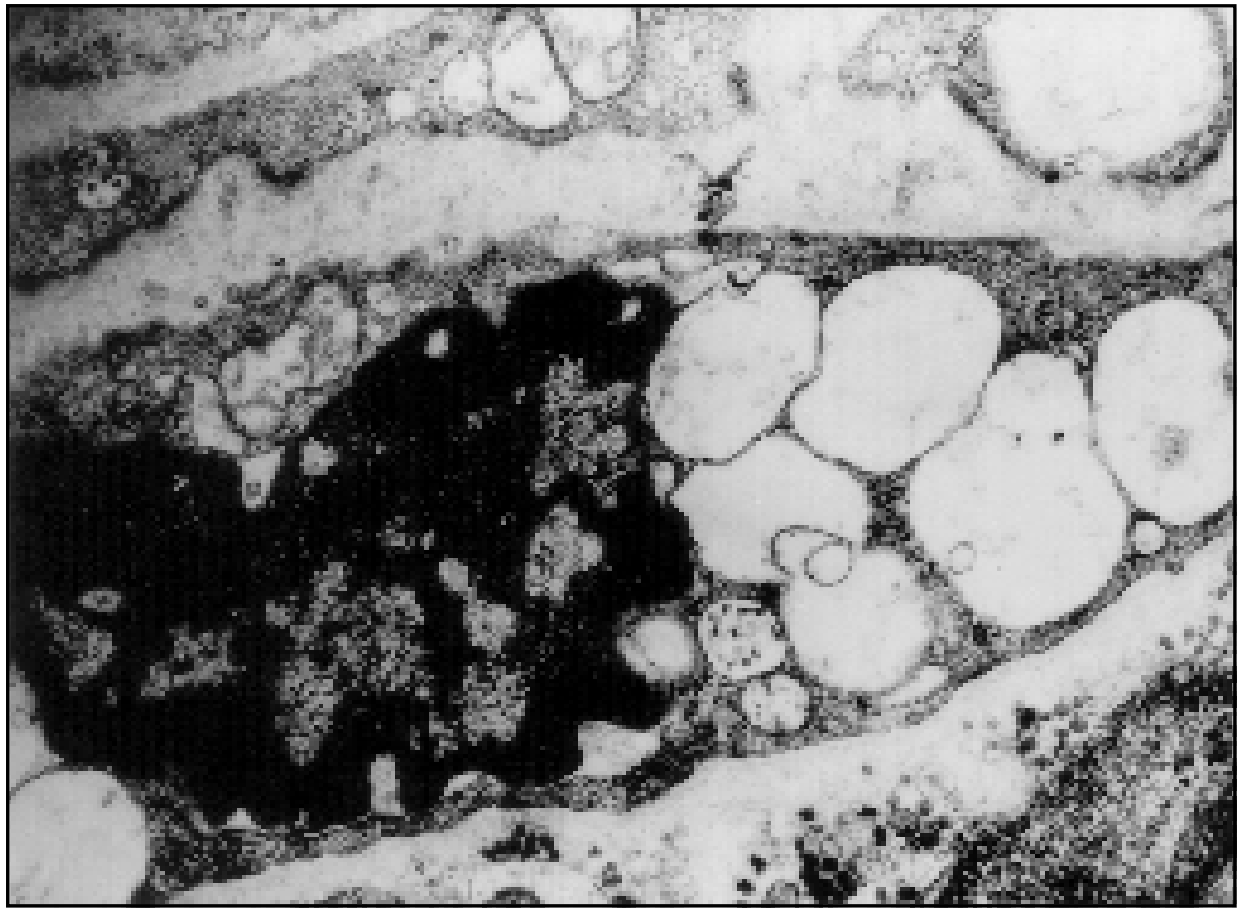

Fig 7. Mucopolissacaridose não identificada bioquimicamente. Vacúolos claros em fibroblasto conjuntival.

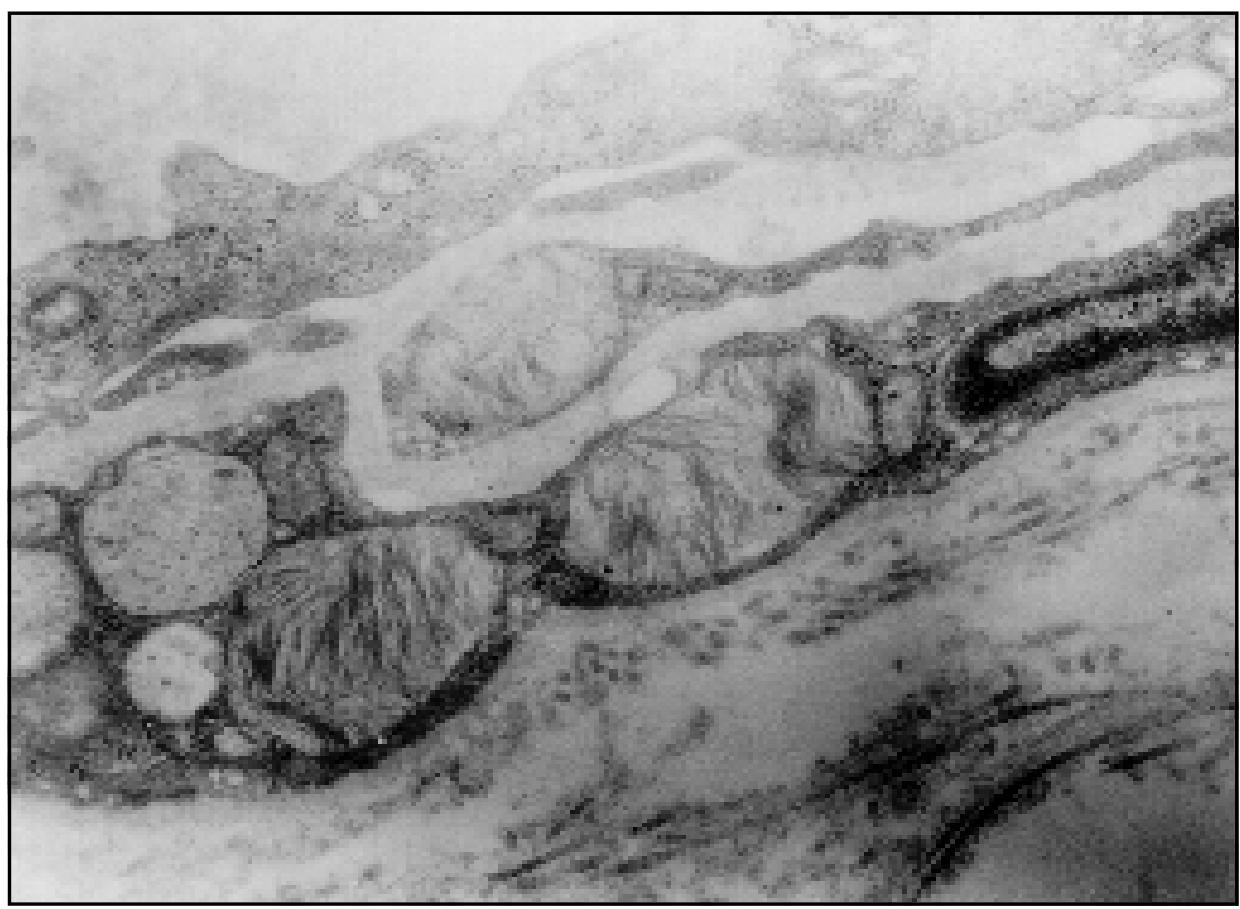

Fig 8. Gangliosidose GM2 (Tipo?). Corpúsculos membrano-citoplasmáticos zebrados em célula endotelial de conjuntiva. 
terminais axonais distróficos (Fig 2) e de inclusões intracitoplasmáticas características (estas visíveis mesmo em microscopia óptica) (Fig 3) são patognomônicos da lipofuscinose neuronal infantil tardia (doença de Bielschowsky) ${ }^{3}$, da distrofia neuroaxonal infantil ${ }^{4,5}$ e da doença de Lafora, respectivamente ${ }^{6}$.

As demais doenças nas quais o exame ultra-estrutural é um importante método diagnóstico são as lisossomopatias, nas quais as características morfológicas do material de depósito intralisossomial resultante de sua não metabolização em razão da deficiência enzimática, variam conforme a entidade ou grupos de entidades afins ${ }^{1,7}$. Em alguns casos, o perfil ultra-estrutural desse material é específico, permitindo o diagnóstico de per si, independentemente de informações clínicas ou da ajuda de outros exames laboratoriais. É o caso dos perfis mielínicos encontrados nas células de Schwann na leucodistrofia metacromática ${ }^{8}$ (Fig 4), dos corpos curvilíneos na doença de Bielschowsky $^{3}$ (Fig 1), dos perfis cristaliformes da doença de Krabbe ${ }^{9,10}$ (Fig 5), dos corpos de Gaucher $^{11}$ ou dos corpos membrano-citoplasmáticos na doença de Niemann-Pick ${ }^{12}$ (Fig 6).

Em outras ocasiões, os achados ultra-estruturais são comuns a grupos de doenças, como os vacúolos claros nas mucopolissacaridoses ${ }^{13,14}$ (Fig 7). Nesses casos, o diagnóstico de certeza necessita da combinação de dados clínicos e/ou laboratoriais com a análise morfológica.

Finalmente, mesmo combinando exame morfológico com dados clínicos e laboratoriais, o diagnóstico de certeza não pode ser feito, pois estes podem ser comuns a mais de uma doença, como ocorre nas mucopolissacaridoses e nas gangliosidoses GM2, nas quais os corpúsculos membranocitoplasmáticos (Fig 8) são comuns a todas suas formas ${ }^{15}$. Aqui, o teste enzimático é fundamental para o diagnóstico exato da entidade.

Em nossa experiência, o exame ultra-estrutural revelou-se de utilidade diagnóstica em 112 casos (23\%). Dada a diversidade dos centros de encaminhamento, é impossível avaliar-se se o fato de $75 \%$ dos casos terem sido negativos se deve a inoportunidade do pedido do exame. Entretanto, em um número certamente não negligenciável dos casos, a própria negatividade do exame deve ter permitido ao clínico afastar a hipótese inicial, abrindo-lhe caminho para outras vias de investigação diagnóstica.

É inegável, portanto, que o exame ultra-estrutural em tecido não cerebral permanece um valioso método diagnóstico das doenças metabólicas do sistema nervoso, mormente onde a análise bioquímica é de difícil execução.

\section{REFERÊNCIAS}

1. Rosemberg S, Campos C. Estudo ultra-estrutural de biópsias de conjuntiva em doenças metabólicas do sistema nervoso. Arq Neuropsiquiatr 1987;45:7-15.

2. Rosemberg S. Neuropediatria. $1^{a}$ Reimpressão. São Paulo: Sarvier, 1995:237.

3. Carpenter S, Karpati G, Andermann K, Jacob JC, Andermann E. The ultrastructural characteristics of the abnormal cytosomes in Batten-Kufs' disease. Brain 1977;100:137-156.

4. Arsenio-Nunes ML, Goutières F. Diagnosis of infantile neuroaxonal dystrophy by conjunctival biopsy. J Neurol Neurosurg Psychiatry 1978;41:511-518.

5. Rosemberg S, Arita FN, Campos C. Distrofia neuroaxonal infantil: diagnóstico em vida por biópsia conjuntival. Arq Neuropsiquiatr 1985;43:48-54.

6. Carpenter S, Carpati G. Sweat gland duct cells in Lafora disease: diagnosis by skin biopsy. Neurology 1981;31:1564-1568.

7. Lyon G, Adams RD, Kolodny EH. Neurology of hereditary metabolic diseases of children. 2.Ed. New York: Mc Graw-Hill, 1996:346.

8. Toga M, Bérard-Badier M, Pinsard N, Gambarelli D, Hassouin J, Tripier MF. Etude clinique, histologique et ultrastructurale de quatre cas de leucodystrophie métachromatique infantile et juvénile. Acta Neuropathol 1972;21:23-38.

9. Lyon G, Jardin L, Aicardi J. Etude au microscope électronique d'un nerf péripherique dans un cas de leucodystrophie de Krabbe. J Neurol Sci 1971;12:263-274.

10. Rosemberg S, Kliemann SE, Arita FN. Doença de Krabbe (leucodistrofia de células globóides): a propósito de cinco observações. Arq Neuropsiquiatr 1992;50:334-342.

11. Hernandez F, Bueno M. Infantile neurological Gaucher's disease in three siblings: an ultrastructural study. Virchows Arch 1973;360:27-32.

12. Anzil AP, Blinzinger K, Mehraein P, Dozic S. Niemann-Pick disease type C: case report with ultrastructural findings. Neuropadiatrie 1973;4:207-225.

13. Arita FN, Campos C. Rosemberg S. Gangliosidose GM1 forma precoce: aspectos clínicos e diagnósticos (A propósito de quatro observações). J Pediat 1985;58:299-307.

14. Arita FN, Rosemberg S. Síndrome de Sanfilippo (mucopolissacaridose III): estudo clínico das formas A e B. Rev Bras Neurol 1988;24:135-138.

15. Terry RD, Weiss M. Studies in Tay-Sachs disease: II. Ultrastructure of the cerebrum. J Neuropathol Exp Neurol 1963;22:18-55. 